\title{
ALGUNAS TENDENCIAS DE LA DIFERENCIACIÓN DEL DERECHO EN CHILE
}

SOME TRENDS IN THE

DIFFERENTIATION OF LAW IN CHILE

Hugo Cadenas ${ }^{1}$

RESUMEN: En este trabajo se aborda el problema de la diferenciación del sistema jurídico partir de un análisis sistémico, tomando el caso de Chile como un ejemplo. Teniendo en cuenta que se trata de una reflexión formal, aunque el análisis se ejemplifica con el caso chileno, muchas de las consideraciones que aquí se presentan se extiendan a otros países de América Latina. El texto presenta los conceptos básicos de la teoría de la diferenciación funcional, se discute el problema de la autonomía de la ley como una condición de diferenciación y se presentan las tendencias, cuestiones y la semántica del proceso producido en Chile. El artículo concluye con reflexiones sobre América Latina.

Palabras Clave: Diferenciación funcional; Luhmann; Derecho; Chile; América Latina

[1]Antropólogo Social, Magíster en Antropología y Desarrollo, profesor en el Departamento de Antropología, Universidad de Chile, Santiago de Chile, e-mail: hcadenas@u.chile.cl 
ABSTRACT: This article addresses the problem of the differentiation of law system based on an systemic analysis of the process in Chile. Since this is a reflection of more formal character, although the analysis is exemplified by the Chilean case, many of the conditions here discussed may be found in other Latin American countries. The article presents the central concepts of the theory of differentiation, is discussed the problem of the autonomy of law as a condition of differentiation and presents trends, problems and semantics of the process in Chile. The article concludes with reflections on Latin America.

Key-words: Functional differentiation; Luhmann; Law; Chile; Latin America.

\section{INTRODUCCIÓN ${ }^{1}$}

Las siguientes reflexiones tienen la intención de formar parte de los estudios de la sociología del derecho. En este sentido, nuestras ideas venideras tendrán como contexto la vasta tradición de la sociología del derecho y sus aplicaciones. En efecto, el presente trabajo intenta aplicar una perspectiva sociológica del derecho para la observación de fenómenos sociales empíricos y, desde allí aportar al conocimiento de la disciplina. Nuestro foco de interés será abordar algunas tendencias en la diferenciación del derecho en Chile. Para atender a este proceso será necesario observar con detenimiento algunos fenómenos transversales que resultan de interés para comprender la deriva específica del derecho en esta región. Este tipo de esfuerzos ha sido escaso en la sociología latinoamericana. Al analizar los procesos de modernización y cambio social (un tema clásico en la sociología latinoamericana) la sociología ha dejado en segundo plano los procesos de cambio jurídico. Si bien resulta comprensi-

[1] Quisiera agradecer a Aldo Mascareño y a Guilherme Brandão por sus observaciones, correcciones y complementos. Este artículo corresponde a una versión, más breve en algunos aspectos y más extensa en otros, respecto de una investigación que aparecerá prontamente en un libro. 
ble que localmente la atención sociológica preste mayor atención a procesos de evidente estratificación social, no aparece justificada la costumbre de reducir estos procesos a meros cambios organizacionales y doctrinarios dentro de la administración de la justicia, en cuyo análisis, acaso, cabe preguntarse por los "efectos" de dichos cambios en la sociedad.

Si se atiende a la tradición de la sociología del derecho, se puede ver que este tipo de enfoques resulta deficitario. Algo que resulta de interés general cuando se intenta caracterizar a la sociología del derecho, es que ésta trató desde sus inicios al derecho desde un punto de vista diferenciado de la Dogmática Jurídica y la Jurisprudencia. Desde sus inicios las ciencias sociales, en cuyo horizonte estuvieron los fenómenos jurídicos, se distanciaron del conocimiento puramente interno del derecho y la búsqueda de la coherencia lógica del mismo. Su propósito fue desarrollar una visión contextualizada y "realista" del derecho en la sociedad. (Baumgartner, 2001; Deflem, 2006; Friedmann, 1962; Kidd, 1938; Krawietz, 1998; Machura, 2001; 2010; Rehbinder, 1972; Tomasic, 1985).

La tradición de la sociología del derecho ha sido fruto de un esfuerzo de largo aliento. Tan solo en la sociología y la antropología social esta tradición tiene más de cien años. Hacia fines del siglo XIX y comienzos del siglo XX se encuentran los primeros esfuerzos por desarrollar una perspectiva sociológica del derecho, las influyentes obras las obras de Sir Henry Maine (2001), H. L. Morgan (1877), Max Weber (1922a; 1922b), Emille Durkheim (2001) y Roscoe Pound (1910), por citar algunos ejemplos, son testigos de esto.

En los años recientes se ha observado una revitalización de la sociología del derecho, junto con una revisión crítica de algunos postulados clásicos, en torno a los cuales las ciencias sociales constituyeron su conocimiento en torno al derecho. Fenómenos como la diferenciación de sistemas sociales autorreferenciales (Luhmann, 1997a), la mundialización de la diferenciación funcional (Stichweh, 1998), el reordenamiento de la política moderna (Willke, 1992) y la globalización del derecho (Teubner, 2005) por mencionar sólo al- 
gunos, presentan problemas a las tradiciones sociológicas y hacen preciso reconceptualizar al derecho y el análisis sociológico que de éste se ha hecho.

Siguiendo estos lineamientos, se hace cada vez más necesario comprender la situación del derecho de la sociedad, sobrepasar la dicotomía (e implícita diferenciación de roles) de "derecho y sociedad", mantenida no sólo por la mayoría de la doctrina positivista del derecho sino también fomentada por la propia sociología del derecho desde al menos la década de los 70's (Baumgartner, 2001). Hoy en día la sociología del derecho se encuentra en un estado de subdesarrollo en América Latina y no son numerosos los trabajos que se han destinado a comprender procesos de cambio social y jurídico. Es usual en los análisis tradicionales del derecho en el contexto de los Estados Nacionales de América Latina el poner un especial énfasis en una "cultura jurídica", la cual explicaría en gran medida -si es que no totalmente- la identidad, tradición, cambios y estabilizaciones del derecho. La convicción acerca de una cultura jurídica "interna" al derecho se encuentra reforzada por la propia sociología y antropología cultural, de las cuales se nutre en parte este enfoque. Ha sido objeto de debates especialmente en la filosofía del derecho y, de manera análoga a este tipo de debates en la antropología social, las conclusiones son diversas y divergentes (Valle, 2001; Fuenzalida, 2002, 2003; Binder, 2007; Squella, 2000). Este debate no ha llevado a la sociología del derecho a buen puerto.

La diferenciación de sistemas debe ser entendida siguiendo un razonamiento formal y estructural, y con esto se hace plausible analizar la diferenciación del derecho en Chile. La diferenciación del derecho aparece en primer lugar como un proceso social, objetivo, temporal y espacial, en el cual se aprecia tanto un aumento de la dependencia como de la independencia del sistema (Luhmann, 1991, p. 250) y en cuya estructuración se conjugan procesos comunicacionales. Si no suponemos que la diferenciación funcional es un estado normativo, que se acepta o rechaza en función de su cercanía al canon luhmanniano, sino que es una estructura con la cual se hace 
posible describir peculiaridades, entonces no se hace preciso justificar normativamente las deficiencias de las estructuras sino el estado regional de comunicaciones diferenciadas en sistemas funcionales.

El objetivo de las presentes reflexiones es abordar algunas tendencias de la diferenciación del derecho en Chile, aunque teniendo en mente siempre la posibilidad de extender este análisis hacia otros países de la región. Nuestra tarea es, sin duda, modesta y apuntará a destacar algunas tendencias presentes que son observables en la diferenciación del derecho y observar desde allí sus semánticas y problemas centrales. En el apartado I abordaremos el problema del cambio social en la teoría de sistemas de Luhmann, los conceptos de diferenciación y evolución y sus rendimientos para la descripción de las transformaciones sociales. En el apartado II atenderemos al concepto de autonomía, el cual aparece dentro de los procesos de diferenciación del derecho y, es en efecto, la cara más visible de la clausura operativa del sistema. En el apartado III se analizan las tendencias principales del proceso de diferenciación, los problemas de referencia que allí emergen y sus semánticas autorreferenciales. Finalmente en el apartado IV se señalan algunas conclusiones producto de las reflexiones anteriores, así como algunas perspectivas para la observación de la diferenciación en Chile y en América Latina.

\section{EL DERECHO EN LA TEORÍA DE LA DIFEREN- CIACIÓN}

El derecho en Chile ha estado afectado durante los últimos años por profundas transformaciones de carácter formal y procedimental. La representación más aguda de estos cambios se ha dado bajo el lema de las reformas. La reforma procesal penal, que iniciada en la década de los noventa, cambió drásticamente el antiguo proceso inquisitorio por un procedimiento más transparente, garantista y equitativo. Este cambio procedimental también ha tenido lugar en otros países de la región con impactos comparativamente más limitados (Witker \& Nataren, 2010). La reforma a los tribunales 
de familia tuvo como objetivo agilizar los procesos en materias de protección jurídica al menor, seguridad jurídica ante el divorcio y casos de violencia intrafamiliar (Casas et al., 2006). Finalmente el cambio en la ley de responsabilidad penal adolescente cambió la manera en que son manejados los problemas de delincuencia juvenil, adaptando las penas a la edad de los inculpados, estableciendo medidas precautorias y de rehabilitación (Hernández Basualto, 2007). Sin duda estas reformas han estado en el centro del debate acerca de su efectividad y racionalidad. No nos ocuparemos de este debate propiamente tal, sino de algunas tendencias que emergen en la diferenciación del sistema del derecho en Chile, no sólo en el contexto de estas importantes transformaciones, sino a nivel general dentro del proceso de diferenciación del sistema. Tenemos como supuesto que el proceso de diferenciación del derecho se ha visto agudizado a partir del cambio político acaecido en los años noventa y que repercutió en cambios en el poder judicial y en las expectativas normativas de la población en Chile. Estas transformaciones políticas han estado presentes en la gran mayoría de los países de la región y han ido acompañadas por otros procesos de modernización. En el caso de Chile la diferenciación de diversas esferas sociales ha ido acrecentándose, a partir de un proceso de modernización cuya tendencia si bien ha sido de carácter económico-liberal, ha plausibilizado de manera paralela la diferenciación de otros sistemas sociales (y sus conflictos). La diferenciación de sistemas sociales autónomos es una expresión directa del proceso de modernización en Chile y sus efectos se extienden desde el Estado como eje institucional, la actividades económicas, pero también en otras comunicaciones como el deporte, la religión, la educación, el medioambiente, etc. La expansión de las comunicaciones a través de redes sociales electrónicas hace acrecentar estas diferenciaciones, complejizan la política, la familia, etc. En todos estos fenómenos se manifiesta la tendencia a la diferenciación de comunicaciones orientadas hacia problemas de referencia específicos. Por estas razones, al tratar el problema de la diferenciación del 
derecho en Chile es preciso conocer primero los fundamentos del concepto de diferenciación.

El concepto de diferenciación es uno de los conceptos clásicos en la teoría social y ha sido empleado con diversos propósitos: para comprender la evolución social desde sus aspectos puramente formales (Spencer, 1912); para describir las consecuencias morales de la creciente división del trabajo en la naciente sociedad industrial (Durkheim, 2001); para comprender la expresión del fenómeno de la "socialización" mediante la distinción forma/contenido (Simmel, 1992); o también las esferas de valor en el capitalismo racional (Weber, 1992b); hasta la especialización funcional de sistemas de acción (Parsons, 1961). En todos estos casos se concibió la modernización social como un proceso de diferenciación (Nassehi, 2011, p. 02). Respecto del derecho, esta idea está presente desde el concepto de diferenciación factual del derecho de Max Weber (1922b, p. 386 y ss.) hasta el razonamiento de Parsons sobre la diferenciación institucional del derecho como mecanismo de integración (1962; 1966, p. 26 y ss; 1971, p. 20 y ss.). En la formulación de Luhmann, esta idea modernizadora del derecho aparece con claridad en sus análisis tempranos sobre las consecuencias de la desdiferenciación (1974) y luego es en cierto modo abandonada. El derecho aparece en dichos análisis tempranos dentro de un proceso más amplio de civilización de expectativas (1974, p. 94 y ss.), las cuales son condición de un orden diferenciado y, junto a los derechos fundamentales, del orden social moderno. En análisis posteriores este diagnóstico del derecho es matizado respecto de las condiciones de diferenciación del derecho como institucionalización, en una vena mucho más parsoniana (2008a, p. 64 y ss.), hasta llegar finalmente al análisis del derecho desde la perspectiva de la sociedad mundial y la diferenciación funcional (1995a). Lo que resulta de sumo interés es que junto a este alejamiento del derecho como condición de modernización de la sociedad, se atestigua asimismo un movimiento hacia la consideración del derecho como un elemento normativo singular, pero no central, en el orden social moderno. 
En este último sentido, el concepto de diferenciación apunta al cambio de forma en la estructura social mediante la repetición de la diferencia entre sistema y entorno dentro del sistema (Luhmann, 1991, p. 22). La diferenciación es un proceso disyuntivo y en algunos casos incluso una anomalía ${ }^{1}$ la cual se ve reforzada por operaciones ulteriores de re-entrada de la forma en la forma. El concepto apunta a que en la formación de sistemas sociales su operación fundamental tiende a generar diferencias, las cuales se refuerzan momento a momento, en cada operación. La diferenciación apunta a la capacidad de transformación social a partir de sus propios condicionantes estructurales y como tal a la formación de una estructura que hace posible a dichas operaciones.

Respecto de los cambios sociales, esta posición conceptual oscila entre una teoría de la evolución dedicada especialmente a los logros evolutivos mediante procesos de variación, selección y re-estabilización (Luhmann 1997a, p. 410 y ss.) y una teoría de la diferenciación orientada a describir los cambios estructurales en el sistema social. En términos generales, es posible afirmar con Stichweh que el problema del cambio social es usualmente tratado por la teoría de sistemas desde el concepto de diferenciación y en segundo lugar mediante el concepto de evolución (Stichweh, 2007, p. 529 y ss.), ${ }^{2}$ mientras que el foco de la teoría de la diferenciación ha estado centrado especialmente en el caso de la diferenciación funcional en la sociedad moderna.

No nos ocuparemos de los logros evolutivos del derecho en Chile a partir un examen histórico de las tres funciones antes descritas, es decir la evolución del sistema por la generalización de las funciones de variación, selección y re-estabilización a partir de las expectativas normativas, los procedimientos de decisión y las formulaciones regulativas del derecho vigente, respectivamente (Luhmann, 1999b, p.16), sino más bien de la forma de diferenciación (Luhmann, 1997a, p. 609) que sirve de trasfondo a la emergencia de

[1] Una "anomalía europea" (europäische Anomalie) señala Luhmann (1995a, p.586).

[2] Stichweh ha llamado la atención especialmente sobre este aspecto. V. Stichweh Op cit. 
tendencias y problemas. No nos ocuparemos de atender a los logros evolutivos del derecho, ni su emergencia como variación, su selección y posterior re-estabilización. El problema evolutivo del derecho se ubica en el plano de las descripciones acerca de estos factores, los cuales si bien resultan de interés para el análisis del derecho, por razones de espacio y profundidad no los atenderemos. En cambio buscaremos los condicionantes en el proceso de diferenciación del sistema del derecho, los factores que indican que la diferenciación del sistema se lleva a cabo operacionalmente y que conducen a una especificación funcional del mismo. Al tratar el tema de la diferenciación desde esta perspectiva, este proceso en el caso del derecho se relaciona con la forma que adopta la diferenciación funcional y, para nuestros análisis, en dimensiones de sentido determinadas. Diferenciación funcional significa a nivel factual $^{l}$ la especificación de problemas de referencia a nivel de sistemas funcionales autónomos, el surgimiento de medios simbólicos para generalizar la aceptación de ofertas comunicacionales a nivel social y la libertad para seleccionar determinadas soluciones frente a otras de manera contingente en un horizonte temporal variable.

Tanto el enfoque de la desdiferenciación episódica del derecho (Mascareño, 2004) como de la constitucionalización simbólica en América Latina (Neves, 1996) comparten el diagnóstico de que la diferenciación funcional adquiere diferentes matices y consecuencias estructurales a partir de la configuración del sistema funcional del derecho. En América Latina estas son condiciones de modernidad periférica, donde la exclusión social generalizada hace aparecer redes de reciprocidad de forma paralela a los sistemas funcionales

[1] El concepto factual como dimensión del sentido corresponde a una traducción controversial al español pues el término en alemán "sachlich" del cual proviene no posee una traducción exacta. En las traducciones existentes se ha empleado este concepto como: objetual o material, a pesar de que el propio Luhmann ha indicado el problema de este tipo de traducción (en este caso al inglés): "At this point in the argument I am at a loss with the English language because there is no equivalent for the German word sachlich. The English words substantial, material, and objective do not precisely correspond to what I actually want to say, so I will proceed with sachlich." (Luhmann 1993b, p.538). Parece más apropiado emplear el término factual en tanto se evita toda referencia a aspectos ontológicos como objetos, sustancias o materiales y se mantiene la referencia a la indicación de la comunicación acerca de la demarcación de temas. 
(Robles, 2000; Mascareño, 2010). Es precisamente por esto que el diagnóstico de la diferenciación funcional debe ser abordado desde todas las dimensiones de sentido y atendiendo tanto a aspectos internos al derecho como a sus relaciones con el entorno. A la par se hace patente que el problema de la diferenciación del derecho no es únicamente un problema de mantenimiento de fronteras, es decir, de autorreferencia, sino también un problema de heterorreferencia, pues para comprender la diferenciación del derecho no basta con atender solamente a los problemas organizacionales (Luhmann 1999a, p. 37).

Para abordar plenamente estos problemas se hace preciso superar una atávica distinción de la sociología del derecho: la diferencia entre ciencia del derecho y sociología del derecho. ${ }^{1}$ Esta distinción formó parte de la fundación de la sociología del derecho y tuvo como propósito establecer una clara frontera respecto de la doctrina jurídica tradicional, especialmente de aquella de tradición kantiana ${ }^{2}$ según la cual el conocimiento del derecho debía de estar basado en aspectos puramente conceptuales y formales. La sociología del derecho diferenció un campo específico al separar este tipo de conocimiento del conocimiento sociológico (empírico). Tanto W. Holmes (1897), Roscoe Pound (1910), Eugen Ehrlich (1989) y Max Weber (1922a; 1922b) compartían esta idea. Las críticas de Kelsen hacia la sociología del derecho apuntaron en gran medida justamente a la plausibilidad de una separación de ese tipo, considerando el objeto de la sociología del derecho como un problema (Kelsen, 1915). Resulta interesante comprobar que para Luhmann esta idea, tan propia de la sociología del derecho, puede ser superada de dos maneras. En primer lugar, reconociendo la relación -weberiana- entre legitimidad y legalidad $^{3}$ pero de manera despegada de un conservadurismo

[1] Esta distinción, aunque relevante, no es universal para la sociología del derecho. Toda la tradición de estudios socio-jurídicos de inspiración marxiana se basa en la forma derecho/ideología, desde el propio Marx, pasando por la escuela de Frankfurt y los estudios crítico-legales. V. Luhmann (2009, p. 247), como panorama general también Tomasic (1985, p. 08 y ss.).

[2] Hay que recordar que para Kant una ciencia empírica del derecho no podía ser más que la cabeza de la fábula de Fedro, una cabeza bella pero sin cerebro (Kant, 1797, p. XXXII).

[3] No sería correcto asimilar la noción de racionalidad weberiana al proceso de diferenciación que 
positivista -como en Kelsen. Vale decir, hacer plausible una relación evolutiva entre el sistema del derecho y otros sistemas sociales. En segundo lugar, mediante la consideración relativa a que la dogmática posee un valor preponderante para el sistema jurídico en su diferenciación interna. La dogmática afianza el código en las decisiones y procedimientos, es una forma de autorregulación del sistema legal mediante autoobservaciones (Luhmann 1995a, p. 387). A través de ella es posible para el sistema observar sus observaciones y distinguir entre norma (como programa) y decisión (como aplicación del código del sistema) y traspasar la contingencia al procedimiento de las decisiones. En ella se definen casos y controversias, junto con criterios de decisión. Estas aclaraciones son muy necesarias, pues para la observación del sistema del derecho se hace preciso no solamente atender a su manifestación "social", lo que se percibe fuera de la organización judicial, sino atender también a su funcionamiento interno, su estructura y semántica.

En suma, la diferenciación del derecho en una comprensión global tiene como supuesto la emergencia de un sistema funcional encargado de producir y tratar el problema específico de las expectativas normativas (Luhmann, 1995a, p. 131) las cuales circulan en el sistema mediante el símbolo de validez. El sistema así diferenciado es capaz de anticipar conflictos y regularlos mediante procedimientos, como una especie de sistema inmunológico (Luhmann 1991, p. 509 y ss.). La legitimación social del derecho, por su parte, viene dada por la vía de acoplamientos políticos como las garantías democráticas y los procesos judiciales (Luhmann, 1983, p. 229 y ss.). El derecho aparece como un sistema social encargado de una función determinada, la cual entraña la paradoja que el sistema es a la vez irreemplazable en su función pero elegible como alternativa funcional. No todos los conflictos han de pasar por el sistema del derecho y sus procedimientos, pero en su función de tratamiento de expectativas normativas válidas éste es irreemplazable. ¿Qué sucede a nivel

destaca Luhmann para el derecho, dado que el derecho como sistema social no se caracteriza por su previsibilidad (aspecto típico de la racionalidad formal del derecho) sino por la contingencia y el riesgo (Cf. Capps \& Palmer Olsen, 2002). 
de las tendencias de su diferenciación?, ¿cuáles son sus problemas y sus semánticas predominantes? Atenderemos a estas y otras cuestiones en los siguientes apartados.

\section{DIFERENCIACIÓN DEL DERECHO: AUTONOMÍA}

El punto de inflexión en el cual parecen converger los diagnósticos sobre la diferenciación del derecho parece apuntar al problema de la autonomía del sistema como eje central. Veremos que este aspecto si bien es relevante es sólo uno de los factores que se conjugan en la diferenciación del derecho. Al tratarse de un problema usualmente tratado en la ciencia del derecho y visto de manera marginal por la sociología del derecho, se hace preciso añadir algunas reflexiones sobre esto. A nivel empírico, el problema de la autonomía tiene la peculiaridad que aparece tanto como expectativas ciudadanas sobre el sistema jurídico, como también en quienes fungen como operadores internos al derecho: jueces, abogados, funcionarios, etc. La discusión en torno a la autonomía del derecho en Chile tiene largo aliento ${ }^{1}$ aunque durante la última década haya cobrado nuevos bríos. Esta discusión se enfoca en una visión tradicional de la autonomía, o más bien de independencia, según la cual se debe fomentar este aspecto en los jueces y abogados (Luhmann, 1995a, p. 63). La relevancia de un concepto de autonomía para describir al sistema jurídico moderno se debe a la especificación del derecho como sistema social con capacidad de establecer una distinción y operar

[1] Ya en 1967, el entonces Presidente de la Corte Suprema Osvaldo Illanes señalaba, a propósito de la autonomía, en este caso, específicamente económica que: "No queda otra solución que este Poder Público tan trascendente en la vida social tenga una autonomía económica. La tiene hoy la Universidad de Chile, con mucha más razón un Poder del Estado. No es, por tanto, una cosa difícil de obtener. Basta la buena voluntad de los otros Poderes. La esperamos con anhelo" (Navarro, 1994, p. 246) El tema se refiere específicamente a los aspectos económicos siendo diversificado únicamente en la década de los noventa, en la que aparece el componente político. En el discurso inaugural del año judicial, en 1990 el presidente de la corte suprema Luis Maldonado "propone la necesidad de establecer una autonomía política - lo que se logra mediante la inamovilidad de los magistrados y mediante un sistema de nombramiento idóneo-, económica —según ya se ha analizado - y funcional - destacando la necesidad de crear una Policía Judicial-." (Navarro, 1994, p. 269). 
de manera unitaria (Luhmann, 1995a, p. 62). La autonomía aparece como consecuencia de la clausura operativa del sistema jurídico.

"De manera diferente a la comprensión usual de la autonomía, distinguimos estrictamente entre cuestiones de dependencia o independencia causal (las que un observador puede juzgar de una u otra manera dependiendo de las causas o efectos que seleccione) y cuestiones de referencia que siempre suponen al sistema como observador." (Luhmann, 1995a, p. 77)

Estas cuestiones de "dependencia o independencia" se refieren a la indicación al sistema o a su entorno como punto de observación, esto es: autorreferencia o heterorreferencia. Hay que señalar que la autonomía de un sistema significa que éste opera como una unidad y que posee una "autolimitación" que hace que la clausura operativa sea un condicionante de esta situación y no su causa (Luhmann, 1995a, p. 63). Estos problemas de dependencia o independencia pueden ser tratados al menos de dos maneras. Desde la función (operación) y desde la codificación (observación), respectivamente.

Como sabemos la función del derecho desde el examen de Luhmann consiste en la estabilización de expectativas normativas, específicamente expectativas normativas congruentemente generalizadas (Luhmann, 2008a, p. 99). En este sentido la autorreferencia desde la perspectiva del sistema se refiere al mantenimiento autolimitado de las expectativas normativas a pesar de su decepción, mientras que la heterorreferencia se refiere a las posibilidades, también autolimitadas, de aprendizaje del sistema, sea éste aprendizaje de tipo doctrinario o legislativo. Esto hace que el derecho esté operativamente clausurado y cognitivamente abierto (Luhmann, 1995a, p. 77, también en 2008, pp. 356-357). ${ }^{1}$

Desde el punto de vista de la decisión jurídica, y decidir es precisamente lo que se espera del derecho, la autorreferencia y la he-

[1] También en Luhmann (2008a, pp.356-357).

84 | Século XXI - Revista de Ciências Sociais, Vol. 2, nº1, p.72-107, jan./jun. 2012

ISSN: $2236-6725$ 
terorreferencia pueden ser vistas también -así lo muestra Luhmanncomo la forma jurisprudencia / legislación (Op cit., p. 302) $)^{1}$, respectivamente. Desde esta perspectiva el sistema jurídico se diferencia en centro y periferia, teniendo en el centro a la jurisprudencia, como decisión jurídica, y en la periferia a la legislación. ${ }^{2}$

Sólo los tribunales se ven en la necesidad de decidir sobre todos los casos posibles que ante éstos se presenten y que ameriten atención legal (Op. cit., p. 310). Sólo en los tribunales se hace posible la convergencia de tres elementos fundamentales para la constitución del derecho: 1) la necesidad de decidir, 2) la libertad de interpretar la norma y 3) la restricción de decidir de manera justa. Solo los tribunales transforman la necesidad en libertad.

Dado que la decisión jurídica está en el centro, la legislación es periferia abierta al entorno y sus demandas. Según Luhmann la tesis jerárquica de que "El juez aplica las leyes obedeciendo las instrucciones del legislador... El tribunal se entiende como una instancia ejecutiva de la competencia legislativa y el método jurídico se entiende como simple deducción" (Op. cit., p. 302) debe ser reemplazada un esquema de centro y periferia que permite resignificar la forma autorreferencia/heterorreferencia. Dado que las decisiones constituyen el elemento central, se ubica en el centro del sistema la organización judicial, es decir, los tribunales y en la periferia la legislación. La periferia, además, sirve de "zona de contacto" (Op. cit., p. 322) con otros sistemas funcionales de la sociedad. En la periferia se pueden absorber las demandas, sin importar si se trata de intereses legales/intereses no legales ya que en ella no existe el imperativo de la decisión. El centro opera con una clausura cognitiva más rígida y en la periferia se presentan las expectativas cognitivas.

La autonomía del sistema se refiere, en suma, a las condiciones en las cuales los procedimientos propiamente jurídicos se ven limitados a partir de su propia clausura operativa. Un sistema

[1] En escritos previos aparece como la forma decisión programante/decisión programada (programmierende/ programmierte Entscheiden) V. Luhmann (1999c, p. 134 y ss).

[2] Esta forma de diferenciación entre centro y periferia también se encuentra en otros sistemas funcionales como la economía y la política (Luhmann, 1995a, pp. 334-335). 
es autónomo no cuando se encuentra en estado de autarquía sino cuando lo que sucede en el sistema está determinado por sus propias estructuras (clausura operativa) y sus límites de influencia son autodeterminados. Los problemas de autonomía en el sistema suceden justamente cuando estos límites son de alguna manera alterados y se pone en juego no solamente la estructura de decisión del sistema sino también su legitimación, en tanto se afecta la generalización de las expectativas acerca de sus procedimientos.

El problema de esta autonomía judicial nos será de interés, aunque nuestras reflexiones apuntan a aspectos de carácter más bien socio-estructural y no centrados únicamente en roles organizacionales del poder judicial. De este modo, no sólo son relevantes los problemas de autonomía decisional sino también los problemas de auto- $y$ heterorreferencia del sistema del derecho, los cuales son multidimensionales a nivel del sentido.

\section{DIFERENCIACIÓN DEL DERECHO EN CHILE: TENDENCIAS}

Al analizar la diferenciación y autonomía del derecho en Chile, y sus problemas específicos, tenemos que aclarar previamente un aspecto clave. Iría aparentemente en contra de un obstáculo epistemológico para la comprensión de la sociedad la consideración de un sistema jurídico nacional, pues supondría que "las sociedades serían unidades regionales territorialmente delimitadas" (Luhmann, 1997a, p.25). Ante esto se hace necesaria una aclaración. La diferenciación funcional como estructura de la sociedad moderna supone mundialización de sistemas y sus operaciones, pero no supone centralización de decisiones. En otras palabras, sociedad mundial no es Estado mundial, escenario que aún hoy día parece improbable (Albert \& Stichweh, 2007). Por ello debemos partir, como toda perspectiva constructivista, desde una diferencia y no desde una unidad. No se trata de considerar la unidad del derecho en Chile (aunque en su operación el derecho suponga su unidad) sino de determinar 
las diferencias que entrañan la forma de la diferenciación en las dimensiones que determina el propio sistema para su operación. Sabemos que la diferenciación funcional no elimina otras formas de diferenciación como la estratificación o la segmentación e incluso en algunos casos las agudiza o las re-especifica de manera funcional al interior de los sistemas (en el caso del derecho por ejemplo mediante la segmentación en Estados o la diferencia centro y periferia entre jurisprudencia y legislación). Estas constelaciones de formas de diferenciación se plasman en lo que se ha denominado múltiples modernidades (Eisenstadt, 2000; Nassehi, 2003a), en las cuales los procesos de modernización tienen acentos particularistas y condiciones estructurales especiales. En el caso de la diferenciación del derecho, nos ocuparemos de rastrear estas especificidades en el nivel de las dimensiones de sentido en tanto intuimos que desde esta perspectiva emergen problemas especiales en la dimensión social y espacial que merecen un examen atento.

Un aspecto controversial en la teoría de sistemas es la consideración de la dimensión espacial para la observación social. En los planteamientos originales de Luhmann esta dimensión está en gran medida ausente debido a que la sociedad como comunicación no posee límites que no estén contenidos en la comunicación. Por esta razón Luhmann reserva el concepto de espacio como un medio para la medición y cálculo de los objetos, relativo a la operación neurofisiológica del cerebro (Luhmann, 1997b, p.179). El espacio -al igual que el tiempo- aparece como medio y forma mediante la distinción $l u$ gar/objeto (Op cit., p.180) y hace visible mediante el cruce de un lado al otro su propia invisibilidad. A raíz de esta reducción -en cierta medida kantiana- del espacio a la observación, se ha mencionado de manera algo crítica que la teoría de sistemas propone una sociedad sin espacio (Schroer, 2005, p. 132 y ss.). Stichweh ha señalado que la dimensión espacial, la cual en la formulación de Luhmann es reducida a la dimensión factual, puede ser incorporada como una dimensión de sentido propia (Stichweh, 2000, p. 187). En efecto, si se toma en consideración la evidencia empírica de que si bien no todos los 
sistemas sociales dan igual relevancia a la distinción espacial de sus comunicaciones y en algunos casos estos problemas son derivados a las organizaciones, en la sociedad moderna el espacio puede ser una dimensión crucial para comprender la diferenciación de determinados sistemas funcionales (Kuhm, 2000; Stichweh, 1998). Mediante las distinciones cerca/lejos y aquí/allá se presenta el espacio en la comunicación (Nassehi, 2003b, p. 222) y la pregunta central acerca de esta dimensión se hace operativa en la comunicación y es empleada en la diferenciación. La pregunta es, en suma, de qué manera el espacio refiere a límites operativos de la sociedad (Nassehi, 2009, p. 447). A partir de esta distinción, se hace patente que el problema de la diferenciación a nivel espacial es resuelto de diversas maneras por los sistemas funcionales. En el caso del derecho, una manera usual de tratar el problema espacial es través de distinciones comunicacionales que apuntan a límites operacionales del sistema. Semánticas como la soberanía, la nacionalidad o la jurisdicción apuntan justamente a definir límites espaciales en el funcionamiento del sistema. De esta manera, y en un nivel político, el derecho se organiza bajo principios estatales con competencias autolimitadas (autónomas) y con ello, define sus fronteras de organización y aplicación.

Además de la dimensión espacial resulta de sumo interés la dimensión social de sentido en el proceso de diferenciación. Es interesante observar que en la sociedad moderna es posible que el derecho pueda enlazar con la política a través de estas dos dimensiones de sentido. En tanto sistemas funcionales derecho y política se encuentran a nivel factual y temporal separados por sus funciones, pero a nivel espacial y social se encuentran acoplados por la delimitación espacial de un territorio para el derecho y la delimitación social de un colectivo para la politica. ${ }^{1}$ Si bien a nivel factual y tem-

[1] Según Armin Nassehi la política hace visible a un colectivo (Nassehi 2009, p. 333) en la dimensión social, idea que se desprende del concepto de política de Luhmann. En efecto, Luhmann adapta casi sin modificaciones el medio simbólico del poder político desde Parsons, es decir, producir decisiones "colectivamente" vinculantes (Parsons, 1963; 1964). La idea del colectivo aparece casi sin discusión en las formulaciones de Luhmann aunque tiene una importancia decisiva para comprender la configuración de la política en la dimensión social de sentido. Es posible que en la adaptación del medio simbólico de Parsons (quién sí había desarrollado formulaciones teóricas acerca de los colectivos) este concepto haya quedado sin revisión posterior. 
poral también se dan acoplamientos (por ejemplo las constituciones políticas o las reglas de administración) en ambos sentidos, social y espacial, la diferenciación de derecho y política son visibles y tienen marcados efectos empíricos. Observar el derecho en Chile implica entonces trazar distinciones desde una perspectiva que demarca territorios y colectivos en comunicaciones y decisiones específicas, pero también implica observar un derecho factual y temporalmente mundializado.

Los análisis sociológicos del derecho en Chile han tenido un marcado acento comparativo, es decir cultural (Luhmann 1999d, p. 47 y ss.) el cual ha estado marcado por el concepto de cultura jurídi$c a{ }^{1}$ En estas coordenadas se ubican los análisis para el caso de Chile de Fuenzalida (2002; 2003), Valle (2001), González Morales (2002) y Squella (2000). En el plano del análisis sistémico, los análisis de la diferenciación funcional en Chile también emplean un enfoque comparativo, especialmente respecto de regiones del mundo más desarrolladas (Mascareño, 2010; Robles, 2000; Rodríguez, 2007). ${ }^{2}$ Así, por ejemplo se destaca que la diferenciación funcional en Chile está marcada por la emergencia de las redes de reciprocidad como alternativa funcional a la orientación por sistemas funcionales (Mascareño, $O p$. cit.; Robles, $O p$. cit. $)^{3}$ de manera diferente a las regiones desarrolladas donde la primacía es hacia sistemas funcionales; los efectos institucionales de las desdiferenciaciones (Mascareño, $O p$. cit.) frente a la diferenciación; o un persistente sustrato cultural familiar (Rodríguez, Op. cit.) ante una sociedad funcionalmente diferen-

[1] El concepto es ampliamente usado en los análisis sociológicos del derecho en Chile y en otros casos. Las elaboraciones conceptuales más usadas son las de Friedman (1969), Cotterrel (1997) y Nelken (2010).

[2] Si se sigue el planteamiento de Luhmann respecto de las observaciones comparativas se podría señalar que este tipo de diagnósticos corresponden a descripciones culturales, en tanto destacan como punto de observación similitudes y diferencias entre regiones mundiales. Sin embargo siguiendo las intenciones de los autores, esto sería adecuado solamente para los análisis de Rodríguez, quien emplea directamente el concepto. Ni Robles ni Mascareño se inclinan por una descripción cultural e incluso el último rechaza este concepto. Cf. Mascareño (2007).

[3] Luhmann señaló, en el caso de la modernización en la Italia meridional, que la formación de redes de reciprocidad este es un aspecto clave para entender los bloqueos a la diferenciación funcional en dicha región (Luhmann, 1995b). Una interesante crítica respecto de este uso del concepto de red en Luhmann se encuentra en Ladeur (2011). 
ciada mundial, etc. Este tipo de análisis es adecuado en tanto señala aspectos comunes y disímiles en la diferenciación funcional mundial, los que permiten destacar aspectos significativos y atienden a la manera en que se producen distorsiones de enlace en las estructuras de los sistemas funcionales. Si bien esta perspectiva atiende acertadamente a problemas fundamentales en la forma de la diferenciación funcional y en la emergencia de modernidades múltiples y específicas, nosotros nos ocuparemos no solamente de estos aspectos estructurales sino también de los problemas de demarcación de fronteras y de autolimitación a nivel espacial y social. Justamente allí donde la diferenciación del sistema deja de ser un problema meramente organizacional (de la administración del derecho) y se acentúa el carácter práctico y empírico del derecho de la sociedad.

Procederemos en el análisis a partir de las dimensiones de sentido señaladas por Luhmann (1991, p. 114 y ss.), de manera de destacar no sólo la complejidad de los problemas de diferenciación, sino también sus diferentes tendencias, problemas de referencia y semánticas autorreferenciales.

En la dimensión factual la diferenciación del derecho en Chile en los últimos años se relaciona directamente con la consecuencia más visible de la clausura operativa, es decir, la tendencia hacia $a u$ tonomía, cuyo desarrollo fue coartado durante la dictadura de Pinochet y retomado como proyecto durante los gobiernos democráticos que le sucedieron (Ruiz-Tagle, 2003). Se observan allí procesos de distinción que actualizan la comunicación jurídica y que, paulatinamente, van orientándose a señalar que dentro del ámbito de la comunicación jurídica todo aquello relevante al derecho puede, no sólo ser objeto de decisión jurídica, sino que los criterios con los cuales se desarrollan dichas distinciones son criterios definidos por el propio sistema jurídico (Mascareño, 2004). El problema de referencia en este sentido es el mantenimiento y defensa de límites operativos del sistema. A nivel semántico (Luhmann, 1993a) se observa que el desarrollo de la diferenciación del derecho va de la mano de una creciente independencia. En dicho nivel resulta adecuado prestar aten- 
ción a procesos de cambio de expectativas, equivalentes funcionales, desviaciones y alteraciones. La tendencia hacia una mayor autonomía ha sido reforzada por los juicios sobre DDHH y las denuncias de corrupción en los últimos años (Skaar, 2003; Mascareño, 2004). Sin duda que es un tema relevante para el derecho, pero es sólo un factor entre otros.

A nivel temporal el derecho también sufre transformaciones, en su aspecto funcional más decisivo, el cual es el tratamiento de expectativas normativas. Es decir, la diferenciación del derecho implica asimismo una diferenciación de las normas y éstas se ubican en la dimensión temporal (Luhmann, 1995a, p. 124-125)1. Las reformas que en el último tiempo se han implementado en Chile han apuntado en gran medida a este factor, pero dentro de un contexto organizacional, y han sido comparativamente exitosas en el concierto latinoamericano (Azócar \& Undurraga, 2005; Witker \& Nataren, 2010, p. 15 y ss.). Como estrategias de modernización del derecho han tomado como aspecto central la aceleración del tiempo (Cf. Rosa, 2003, p. 27 y ss.) en los procesos como elemento e indicador de operatividad y eficiencia, la cual también funge como una semántica dominante. Las decisiones dentro del sistema judicial poseen ahora tiempos de ejecución más estrictos, en tanto el tiempo es considerado un recurso limitado. El horizonte temporal del derecho en estos casos se restringe, mientras que la producción normativa aumenta ostensiblemente. Esto conlleva a que el problema de referencia en este plano sea la congruencia de las decisiones en el contexto de las transformaciones.

Por su parte, el derecho en Chile en su trasfondo espacial se ha hecho permeable, de la mano de los procesos de penetración de competencias del derecho internacional (Mereminskaya \& Mascareño, 2006) y la globalización de regímenes jurídicos (Teubner, 2002). En los diversos niveles internos de organización del derecho las referencias espaciales una vez invisibles se hacen visibles a la luz de

[1] Este es un aspecto clave respecto del carácter de las normas frente a posiciones tradicionales. Para Habermas (1987) por ejemplo, las normas deben ubicarse -al igual que para Parsons y Durkheim- en un nivel moral. Cf. Cadenas (2006). 
las diversas competencias jurídicas o colisiones jurídicas existentes. Lo que antes podía ser decidido bajo el contexto del imperio de la soberanía hoy es objeto de controversias y disputas, en las cuales se problematiza el cómo se distingue un derecho en un trasfondo espacial y cómo se determinan las competencias específicas mediante decisiones. Por este motivo el problema de referencia en esta dimensión es la operatividad del derecho en fronteras jurisdiccionales, pues el trasfondo espacial del derecho ha sido alterado. La dimensión espacial es un elemento clave en la diferenciación del derecho pues éste, a diferencia de otros sistemas funcionales como la ciencia o la economía, ${ }^{1}$ reconoce especial relevancia al problema de las fronteras en las cuales el derecho puede operar de manera estructuralmente coherente. En esta dimensión el derecho se asienta en problemas de referencia espacial que ganan en complejidad en sus operaciones y ponen en juego sus diferencias constitutivas. La semántica predominante es la relativa a la globalización del derecho y sus consecuencias para el funcionamiento del sistema (Gessner, 2010; Günther, 2003). En América Latina esto no está exento de problemas, pues el rol que juega el derecho internacional en la legislación nacional es mayoritariamente secundario y objeto de controversias a la par del rol cada vez más supranacional que juega el derecho (López-Ayllón, 2010, p.141 y ss.).

En la dimensión social de sentido los mundos de la vida de las personas se van juridificando (Habermas, 1982) y creando nuevos espacios de inclusión, tales como las regulaciones de familia (Casas et al., 2006), los problemas medioambientales (Dourojeanni \& Jouravlev, 1999), la vinculación religiosa, educativa o ética (Bascuñán Rodríguez, 2004). En la dimensión que relaciona a alter y ego en la comunicación, el derecho penetra paulatinamente y de maneras que permean la vida cotidiana de las personas. El problema de referen-

[1] En estos sistemas si bien se aprecia una mundialización de conocimientos y teorías, en el caso de la ciencia) o la desterritorialización de las operaciones económicas marcadas por el medio del dinero siguen teniendo como relevancia aspectos espaciales. La economía sigue estando anclada en conceptos como propiedad y la ciencia distingue, a pesar de su mundialización, entre centros y periferias de producción científica. 
cia en este nivel es claramente la clarificación de las competencias sobre relaciones sociales de diversa índole. Estos efectos se visualizan tanto a nivel de regulaciones civiles como penales, vale decir, tanto en las relaciones que tienen las personas entre sí, así como las vinculaciones con el Estado. ${ }^{1}$ La diferenciación del sistema en este nivel ha hecho que en Chile incluso los movimientos sociales de los últimos años han tenido un marcado acento juridificador, ya sea para derogar o proponer nuevos cuerpos legales, como es el caso de diversos movimientos educativos de las últimas décadas (Bellei et al., 2010). En todos estos casos el indicador semántico es la inclusión en el derecho, aunque veremos que estos procesos también pueden darse a través de consecuencias paradojales.

Tabla 1: Tendencias, problemas y semánticas de diferenciación del derecho en Chile a través de las dimensiones de sentido.

\begin{tabular}{|c|c|c|c|c|}
\hline & \multicolumn{4}{|c|}{ Dimensiones de sentido } \\
\hline & Factual & Temporal & Espacial & Social \\
\hline $\begin{array}{l}\text { Tendencias } \\
\text { estructurales }\end{array}$ & Autonomía & Aceleración & Permeabilidad & Juridificación \\
\hline $\begin{array}{l}\text { Problemas de } \\
\text { referencia }\end{array}$ & $\begin{array}{l}\text { Mantenimiento } \\
\text { y defensa de lími- } \\
\text { tes operativos }\end{array}$ & $\begin{array}{l}\text { Congruencia de } \\
\text { las decisiones }\end{array}$ & $\begin{array}{c}\text { Operatividad en } \\
\text { fronteras jurisdic- } \\
\text { cionales }\end{array}$ & $\begin{array}{c}\text { Competencias } \\
\text { sobre relaciones } \\
\text { sociales }\end{array}$ \\
\hline $\begin{array}{l}\text { Correlatos } \\
\text { semánticos } \\
\text { autorreferenciales }\end{array}$ & Independencia & Eficiencia & Globalización & Inclusión \\
\hline
\end{tabular}

En la tabla anterior hemos presentado de manera sucinta los ejes de nuestros análisis sobre las tendencias de la diferenciación del derecho en Chile. Sin lugar a dudas estas reflexiones son de carácter exploratorio y apuntan más bien a definir líneas de investigación futuras sobre estos temas. Sin embargo, a pesar del pesimismo reinante tanto en la sociología como en las ciencias jurídicas sobre el papel del derecho en la modernización, es plausible orientar la

[1] En este contexto de juridifcación una interesante investigación acerca de la capacidad de la persona para tomar decisiones, desde un punto de vista médico y legal se encuentra en Bórquez et al. (2007). 
investigación hacia aspectos globales y comparativos del derecho en nuestra región y no detener las reflexiones al constatar que se han defraudado ciertas expectativas normativas (por ejemplo, que fallen los modelos explicativos).

\section{CONCLUSIONES}

En el análisis precedente hemos presentado aspectos generales de la diferenciación del derecho en Chile en las últimas décadas a partir de un análisis multidimensional. Hemos destacado las tendencias de la diferenciación, sus problemas de referencia y las semánticas asociadas a estos procesos.

Finalmente quisiéramos señalar algunos aspectos que resultan relevantes a la hora de extender estas reflexiones hacia otras regiones. En América Latina el estado de los sistemas jurídicos es bastante desigual. Si bien toda la región comparte ciertamente una tradición jurídica continental muy marcada, las diferencias en el desarrollo de cada orden jurídico nacional son muy variables. A pesar de esto y siguiendo una antigua costumbre sociológica, es posible de pensar elementos estructurales similares entre estos regímenes jurídicos nacionales, aunque las temporalidades y causalidades sean ciertamente disímiles.

Quizás el punto donde más acentuadamente se observa una tendencia común en la región es respecto de la tendencia hacia la autonomía. Solamente Colombia puede señalar que desde 1957 su sistema del derecho goza de plena autonomía, aunque ésta sea obra de la junta militar de aquella época, y que con altos y bajos éstas se ha reactivado en los ochentas (Uprimy et al., 2003, p. 242). Aunque por otro lado el país haya vivido, entre 1949 y 1991 más de 32 años bajo una situación de legalidad marcial. Sin embargo no fue el problema de la autonomía o el excesivo uso de los Estados de excepción lo que motivó las reformas en Colombia, sino la corrupción y el narcotráfico. La corrupción, también, sumado al clientelismo derivado de la democracia de "punto fijo" son señaladas como las causas de las re- 
formas legales en Venezuela en los noventas (Pérez Perdomo, 2003, p. 713). Las reformas constitucionales de 1994 en México tuvieron como propósito también aumentar la independencia judicial (Báez Silva, 2010, p. 224). En Brasil se puede señalar que la constitución de 1988, muy orientada hacia los DDHH y la participación ciudadana, también apoyó esta tendencia general (Botelho Junqueira, 2003, p. 128 y ss.). En Argentina, finalmente, con la creación del Consejo de la Magistratura, gracias a la Constitución de 1994, se reforzó también esta tendencia (Bergoglio, 2003, pp. 52-53). Las semánticas son similares también, pues la autonomía se trata usualmente como un problema de "independencia judicial".

La tendencia hacia la aceleración en las decisiones y los problemas de congruencia son compartidos por todos los sistemas jurídicos nacionales que han sufrido reformas en los últimos años. Hay que recordar que sólo a nivel de reformas procesales desde 1991 Argentina, Guatemala, Costa Rica, El Salvador, Venezuela, Chile, Paraguay, Bolivia, Ecuador, Nicaragua, Honduras, República Dominicana, Colombia, Perú, México, Brasil y Panamá (Witker \& Nataren, 2010, p. 06) han experimentado reformas en dichos sistemas. En este sentido es sintomático que en todos estos casos la orientación de estas reformas en su nivel organizacional haya tenido como elemento principal la orientación temporal, aspectos sobre el cual estas reformas han medido su eficacia.

A nivel social, la juridización de las relaciones sociales ha sido atestiguado desde los años noventas también en México (López Ayllón \& Fix-Fierro, 2003, p. 503 y ss.) respecto de la creciente injerencia del derecho en la vida de las personas. Situaciones similares se han analizado en Brasil, gracias a la constitución de 1988 que fomenta la participación ciudadana (Botelho Junqueira, 2003, p. 144 y ss.) y en Colombia de manera paradojal -como creciente visibilidad y relevancia social, pero desinterés generalizado- (Uprimy, 2003, pp. 231-234). En cualquier caso han sido en parte los cambios institucionales en el derecho los que han fomentado esta creciente relevancia social del mismo. Esto ha traído consigo además crecientes deman- 
das sociales sobre justicia al sistema. Hay que recordar que las instituciones legales en América Latina gozan de sospechas fundadas y más de un tercio de la población cree justificado pasarlas por alto en caso de necesidad (Latinobarómetro, 2010). En este contexto la tendencia hacia una mayor juridización en la región puede ser vista de manera menos pesimista.

Finalmente respecto de la tendencia a la permeabilidad el derecho en América Latina ha estado paulatinamente en apertura hacia el derecho internacional. Esta tendencia se observa en toda la región. Hay que recordar que las relaciones entre el derecho nacional y el derecho internacional son diversas en la región. En Argentina, México, Ecuador y Nicaragua sus constituciones establecen, de manera explícita o implícita, la primacía del derecho constitucional por sobre el internacional. En Perú, el artículo 101 de su constitución señala que "los tratados internacionales celebrados por el Perú con otros Estados, forman parte del derecho nacional. En caso de conflicto entre el tratado y una ley, prevalece el primero". Esta situación es similar en Costa Rica, Salvador y Honduras. En Perú y en Chile las constituciones otorgan un estatus "cuasi" constitucional a las normas internacionales en materia de derechos humanos. (López-Ayllón, 2010, pp. 141-142). La apertura hacia el derecho internacional ha sido favorecida en gran medida por la globalización económica y la extensión de las relaciones internacionales. Este fenómeno es relativamente reciente en la región. En México, por ejemplo, esta apertura se observa desde los noventas, pues al menos hasta fines de los años ochentas este país se encontraba más bien cerrado al derecho internacional (López Ayllón \& Fix-Fierro, 2003, p. 582), una situación similar sucede en otros países de la región. Además de esta relación entre derecho nacional/internacional emergen regímenes jurídicos espontáneos (Teubner, 2002; 2005) los cuales aparecen de manera "híbrida" (Gessner, 2010, p. 99) entre el derecho nacional y el internacional, como normas comerciales, técnicas o deportivas (Mascareño \& Mereminskaya, 2006). En todas estas transformacio- 
nes se aprecia una permeabilidad mayor, si se quiere, una tolerancia mayor hacia una pluralidad normativa creciente.

En definitiva, en estas tendencias de diferenciación que hemos descrito, pueden señalar interesantes vías de investigación para una sociología del derecho que se abre a la descripción de la operatividad social. Esta nueva praxis de observación, permite delinear al derecho como una poiesis social (Arnold, 2006) específica, contingente y temporalmente inestable. De este modo la sociología del derecho puede hacer justicia a sus propósitos y sus propios conceptos.

\section{REFERENCIAS}

ALBERT, M.; STICHWEH, R. (Eds.). Weltstaat und Weltstaatlichkeit. Beobachtungen globaler politischer Strukturbildung. Wiesbaden: VS Verlag für Sozialwissenschaften, 2007.

ARNOLD, M. Lineamientos para un programa sociopoiético de investigación. In: FARIAS, I.; OSSANDON, J. (Eds.). Observando Sistemas. Nuevas apropiaciones y usos de la teoría de Niklas Luhmann. Santiago de Chile: RIL, 2006, pp. 219-240.

AZÓCAR, M. J.; UNDURRAGA, B. Reforma Procesal Penal y Seguridad Ciudadana: Análisis de cuatro indicadores. Santiago de Chile: Revista Paz Ciudadana, 2005.

BÁEZ SILVA, C. Cambio Político y Poder judicial en México. In: CABALLERO JUÁREZ, J. A. CONCHA CANTÚ, H. A. FIX-FIERRO, H.; IBARRA PALAFOX, F. (Eds.). Sociología del derecho. Culturas y Sistemas Jurídicos Comparados. Volumen I. Globalización y derecho, justicia y profesión jurídica. México D. F.: Universidad Nacional Autónoma de México, 2010, pp. 209-238.

BASCUÑÁNRODRÍGUEZ, A. La píldora del día después ante lajurisprudencia. Estudios Públicos, nº. 95, pp. 43-89, 2004. 
BAUMGARTNER, M. The sociology of law in the United States. The American Sociologist, Vol. 32, no. 2, pp. 99-113, 2001.

Bellei, C., CONTRERAS, D. \& VALENZUELA, J. P. (Eds.). Ecos de la revolución pingüina. Avances, debates y silencios en la reforma educacional. Santiago de Chile: UNICEF-Universidad de Chile, 2010.

BERGOGLIO, M. I. Argentina: Los efectos de la institucionalización democrática. In: FIX-FIERRO, H., FRIEDMAN, L. \& PÉREZ PERDOMO, R. (Eds.). Culturas jurídicas latinas de Europa y América en tiempos de globalización. México D. F.: Universidad Nacional Autónoma de México, 2003. (pp. 27-115).

BINDER, A. La cultura jurídica, entre la innovación y la tradición. Congreso Federal de Justicia Penal. Anais... Buenos Aires: [s.n.]. , 2007.

BLOCH, E. Erbschaft dieser Zeit. Frankfurt a. M.: Suhrkamp, 1973.

BÓRQUEZ, E. G.; RAINERI, G.; HORWITZ, N.; HUEPE, G. La noción de capacidad de la persona para tomar decisiones, en la práctica médica y legal. Rev. méd. Chile, no. 135, pp. 1153-1159, 2007.

BOTELHO JUNQUEIRA, E. Brasil: Obstáculos en el camino hacia una justicia total. In: FIX-FIERRO, H., FRIEDMAN, L. \& PÉREZ PERDOMO, R. (Eds.). Culturas jurídicas latinas de Europa y América en tiempos de globalización. México D. F.: Universidad Nacional Autónoma de México, 2003. (pp. 117-194).

CADENAS, H. Derecho y Sociedad: ¿Es posible la integración social mediante el derecho? In: FARIAS, I.; OSSANDON, J. (Eds.). Observando Sistemas. Nuevas apropiaciones y usos de la teoría de Niklas Luhmann. Santiago de Chile: RIL, 2006. (pp. 263-281).

CAPPS, P.; OLSEN, H. P. Legal autonomy and reflexive rationality in complex societies. Social \& Legal Studies, Vol. 11, nº. 4, pp. 547-567, 2002. 
CASAS, L. DUCE, M. MARÍN, F. RIEGO, C.; VARGAS, M. El funcionamiento de los nuevos Tribunales de Familia: Resultados de una investigación exploratoria. Santiago de Chile: CEJA, [s.n.], 2006.

COTTERREL, R. The concept of legal culture. In: NELKEN, D. (Ed.). Comparing legal cultures. Aldershot, Hants, England and Brookfield, Vt.: Dartmouth, 1997.

DEFLEM, M. Jurisprudencia sociológica y sociología del derecho. Opinión Jurídica, Vol. 5, n. 10, pp.107-119, 2006.

DURKHEIM, E. La división del Trabajo Social. Madrid: Akal, 2001.

DOUROJEANNI, A., \& JOURAVLEV, A. El Código de Aguas de Chile: entre la ideología y la realidad. Santiago de Chile: CEPAL, 1999.

EHRLICH, E. Grundlegung der Soziologie des Rechts. Berlin: Duncker \& Humblot, 1989.

EISENSTADT, S. N. Multiple modernities. Daedalus, Vol. 129, nº. 1, pp. $1-29,2000$.

FRIEDMAN, L. M. Legal Culture and Social Development. Law \& Society Review, Vol. 4, n. 1, pp. 29-44, 1969.

FRIEDMANN, W. Sociology of Law. Current Sociology, Vol. 11, n. 1, pp. $1-16,1962$.

FUENZALIDA FAIVOVICH, E. La cultura jurídica chilena: Una cultura tensionada por la sucesión de las generaciones y el empuje de la cultura jurídica transnacional. Anuario de Filosofía Jurídica y Social, no. 20, pp. 323-336, 2002.

FUENZALIDA FAIVOVICH, E. Derecho y cultura jurídica en Chile (19741999). In: FIX FIERRO, H. FRIEDMAN, L.; PÉREZ PERDOMO, R. (Eds.). Culturas jurídicas latinas de Europa y América en tiempos de Globalización. México D. F.: Instituto de Investigaciones Jurídicas de la Universidad Nacional Autónoma de México, 2003. (pp. 195-230). 
GESSNER, V. Comparación jurídica y pluralismo jurídico global. In: CABAllero JuÁreZ, J. A. CONCHA CANTÚ, H. A. FIXFIERRO, H.; IBARRA PALAFOX, F. (Eds.). Sociología del derecho. Culturas y Sistemas Jurídicos Comparados. Volumen I. Globalización y derecho, justicia y profesión jurídica. México D. F.: Universidad Nacional Autónoma de México, 2010. (pp. 95-124).

GONZÁLEZ MORALES, F. Algunas observaciones sobre cultura judicial y enseñanza del derecho en Chile. Anuario de Filosofía Jurídica y Social, n'. 20, pp. 373-393, 2002.

GÜNTHER, K. Pluralismo jurídico y Código Universal de la Legalidad: la globalización como problema de Teoría del Derecho. Anuario de Derechos Humanos. Nueva Época, Vol. 4, pp. 225-257, 2003.

HABERMAS, J. Theorie des kommunikativen Handelns. Band 2. Zur Kritik der funktionalistischen Vernunft. Frankfurt a.M.: Suhrkamp, 1982.

Wie ist Legitimität durch Legalität möglich? Kritische Justiz, Vol. 20, no. 1, pp. 1-16, 1987.

HERNÁNDEZ BASUALTO, H. El Nuevo Derecho Penal de Adolescentes y la necesaria revisión de su "Teoría Del Delito". Revista de Derecho (Valdivia), Vol. 20, no. 2, pp. 195-207, 2007.

KANT, I. Metaphysische Anfangsgründe der Rechtslehre. Königsberg: Friedrich Nicolovius, 1797.

KELSEN, H. Eine Grundlegung der Rechtssoziologie. Archiv für Sozialwissenschaft und Sozialpolitik, no. 39, pp. 839-876, 1915.

KIDD, A. M. Review: Fundamental Principles of the Sociology of Law by Eugen Ehrlich. Columbia Law Review, Vol. 38, n. 2, pp. 383-386, 1938.

KRAWIETZ, W. El concepto sociológico del derecho. Doxa: Cuadernos de Filosofía del Derecho, no. 5, pp. 253-274, 1998. 
KUHM, K. Raum als Medium gesellschaftlicher Kommunikation. Soziale Systeme, Vol. 6, nº. 2, pp. 321-348, 2000.

LADEUR, K.-H. Die Netzwerke des Rechts. In: BOMMES, M.; TACKE, V. (Eds.). Netzwerke in der funktional differenzierten Gesellschaft. Wiesbaden: VS Verlag für Sozialwissenschaften, 2011. (pp. 143-171).

LATINOBARÓMETRO. Disponível em: < www.latinobarometro.org/>. Acesso en: 24 jul. 2011.

LÓPEZ-AYLLÓN, S. Tribunales, Seguridad Jurídica y Globalización. In: CABAllero JuÁreZ, J. A. CONCHA CANTÚ, H. A. FIXFIERRO, H.; IBARRA PALAFOX, F. (Eds.). Sociología del derecho. Culturas y Sistemas Jurídicos Comparados. Volumen I. Globalización y derecho, justicia y profesión jurídica. México D. F.: Universidad Nacional Autónoma de México, 2010. (pp. 125-161).

LUHMANN, N. Grundrechte als Institution. Berlin: Duncker \& Humblot, 1974.

. Legitimation durch Verfahren. Frankfurt a.M.: Suhrkamp, 1983.

. Soziale Systeme. Grundriß einer allgemeinen Theorie. Frankfurt a.M.: Suhrkamp, 1991.

Gesellschaftliche Struktur und Semantische Tradition. In: LUHMANN, N. (Ed.). Gesellschaftsstruktur und Semantik. Studien zur Wissenssoziologie der modernen Gesellschaft, Band 1. Frankfurt a.M.: Suhrkamp, 1993a. (pp. 9-71).

- Ecological communication: Coping with the unknown. Systems Practice, Vol. 6, n. 5, pp. 527-539, 1993 b. . Das Recht der Gesellschaft. Frankfurt a.M.: Suhrkamp, 1995a. Kausalität im Süden. Soziale Systeme, Vol. 1, no. 1, pp. 7-28, $1995 b$. 
Die Gesellschaft der Gesellschaft. Frankfurt a.M: Suhrkamp, $1997 \mathrm{a}$.

. Die Kunst der Gesellschaft. Frankfurt a.M.: Suhrkamp, $1997 b$.

LUHMANN, N. Ausdifferenzierung des Rechtssystems. In: LUHMANN, N. (Ed.). Ausdifferenzierung des Rechts: Beiträge zur Rechtssoziologie und Rechtstheorie. Frankfurt a.M.: Suhrkamp, 1999a. (pp. 35-52).

. Evolution des Rechts. In: LUHMANN, N. (Ed.). Ausdifferenzierung des Rechts: Beiträge zur Rechtssoziologie und Rechtstheorie. Frankfurt a.M.: Suhrkamp, 1999b. (pp. 11-34).

. Positivität des Rechts als Voraussetzung einer modernen Gesellschaft. In: LUHMANN, N. (Ed.). Ausdifferenzierung des Rechts: Beiträge zur Rechtssoziologie und Rechtstheorie. Frankfurt a.M.: Suhrkamp, 1999c. (pp. 113-153).

. Kultur als historischer Begriff. In: LUHMANN, N. (Ed.). Gesellschaftsstruktur und Semantik. Studien zur Wissenssoziologie der modernen Gesellschaft, Band 4. Frankfurt a.M.: Suhrkamp, 1999d. (pp. 31-54).

Rechtssoziologie. Wiesbaden: VS Verlag für Sozialwissenschaften, 2008a.

Positives Recht und Ideologie. In: LUHMANN, N. (Ed.). Soziologische Aufklärung 1: Aufsätze zur Theorie sozialer Systeme. Wiesbaden: VS Verlag für Sozialwissenschaften, 2009. (pp. 224-255).

MACHURA, S. German sociology of law. The American Sociologist, Vol. 32, n'. 2, pp. 41-60, 2001.

. Rechtssoziologie. In: KNEER, G. \& SCHROER, M. (Ed.). Handbuch Spezielle Soziologien. Wiesbaden: VS Verlag für Sozialwissenschaften, 2010. (pp. 379-392). 
MAINE, H. S. El Derecho Antiguo. Guadalajara: Editorial Campo Raso, 2001.

MASCAREÑO, A. Sociología del Derecho (Chileno y latinoamericano). Persona y Sociedad, Vol. XVIII, no. 2, pp. 63 - 94, 2004.

. Sociología de la cultura. La deconstrucción de lo mapuche. Estudios Públicos, n. 105, pp. 61-112, 2007.

. La trayectoria de la diferenciación funcional en la región latinoamericana. In: MASCAREÑO, A. (Ed.). Diferenciación y Contingencia en América Latina. Santiago de Chile: Ediciones Universidad Alberto Hurtado, 2010. (pp. 85-119).

MEREMINSKAYA, E. La desnacionalización del derecho y la formación de regímenes globales de gobierno. Sesquicentenario del Código Civil de Andrés Bello: Pasado, presente y futuro de la codificación, Tomo II. Santiago de Chile: Lexis-Nexis, 2006.

MORGAN, L. H. Ancient Society. Or Researches in the Lines of Human Progress from Savagery through Barbarism to Civilization. London: MacMillan \& Company, 1877.

NASSEHI, A. Geschlossenheit und Offenheit. Studien zur Theorie der modernen Gesellschaft. Frankfurt a.M.: Suhrkamp, 2003a.

Welten in der Weltgesellschaft: Die Gegenwart einer Gesellschaft. In: NASSEHI, A. (Ed.). Geschlossenheit und Offenheit. Studien zur Theorie der modernen Gesellschaft. Frankfurt a.M.: Suhrkamp, 2003b. (pp. 188-228).

Der soziologische Diskurs der Moderne. Frankfurt a.M.: Suhrkamp, 2009.

. La teoría de la diferenciación funcional en el horizonte de sus críticas. Revista Mad, no. 24, pp. 01-29, 2011.

NELKEN, D. Repensando en concepto de cultura jurídica. In: CABALLERO JUÁREZ, J. A. CONCHA CANTÚ, H. A. FIX-FIERRO, H.; IBARRA 
PALAFOX, F. (Eds.). Sociología del derecho. Culturas y Sistemas Jurídicos Comparados. Volumen II: Regulación, cultura jurídica, multiculturalismo, pluralismo jurídico y derechos humanos. México D. F.: Universidad Nacional Autónoma de México, 2010. (pp. 137-175).

NEVES, M. Constitucionalização simbólica e desconstitucionalização fática: mudança simbólica da Constituição e permanência das estruturas reais de poder. Brasília, Vol. 33, nº 132, pp. 321-330, 1996.

PARSONS, T. An Outline on the Social System. In: PARSONS, T. SHILS, E. NAEGELE, K. D.; PITTS, J. R. (Eds.). Theories of Society. Vol. 1. Glencoe, Illinois: The Free Press, 1961. (pp. 30-79).

The Law and Social Control. In: EVAN, W. M. (Ed.). Law and Sociology. Exploratory Essays. New York: The Free Press of Glencoe, 1962. (pp. 56-72)).

On the Concept of Political Power. Proceedings of the American Philosophical Society, no. 107, pp. 232-262, 1963.

Evolutionary Universals in Society. American Sociological Review, Vol. 29, no. 3, pp. 339-357, 1964.

. Societies: Evolutionary and Comparative Perspectives. Englewood Cliffs, N.J.: Prentice-Hall, 1966.

The system of modern societies. Englewood Cliffs, N.J.: Prentice-Hall, 1971.

PÉREZ PERDOMO, R. Venezuela 1958-1999: El derecho en una democracia renqueante. In: FIX-FIERRO, H., FRIEDMAN, L. \& PÉREZ PERDOMO, R. (Eds.). Culturas jurídicas latinas de Europa y América en tiempos de globalización. México D. F.: Universidad Nacional Autónoma de México, 2003, (pp. 639-722).

POUND, R. Law in Books and Law in Action. American Law Review, Vol. 44, pp. 12-36, 1910. 
REHBINDER, M. The sociology of Law. Current Sociology, Vol. 20, nº. 3, pp. 13-48, 1972.

ROBLES, F. El Desaliento Inesperado de la Modernidad. Molestias, Irritaciones y Frutos Amargos de la Sociedad del Riesgo. Santiago de Chile: RIL Editores, 2000.

RODRÍGUEZ, D. Communication, Confidence and Trust: Functional Differentiation in Chile. Soziale Systeme, Vol. 13, n. 1+2, pp. 209-220, 2007.

ROSA, H. Social Acceleration: Ethical and Political Consequences of a Desynchronized High-Speed Society. Constellations, Vol. 10, nº. 1, pp. 3-33, 2003.

RUIZ-TAGLE, P. La tesis de la doble pluralidad: jueces y democracia el caso de la transición chilena 1990-2002. In: OROZCO HENRÍQUEZ, J. J. VÁZQUEZ, R.; MALEM SEÑA, J. F. (Eds.). La función judicial. Ética y democracia. Barcelona: Gedisa, 2003.

SCHROER, M. Räume, Orte, Grenzen: Auf dem Weg zu einer Soziologie des Raums. Frankfurt a. M.: Suhrkamp, 2005.

SIMMEL, G. Über soziale Differenzierung. Gesamtausgabe Band 2. Frankfurt a. M.: Suhrkamp, 1992.

SKAAR, E. Independencia judicial y política de derechos humanos en Argentina y Chile. In: BURGOS S., G. (Ed.). Independencia Judicial en América Latina. ¿De quién? ¿Para qué? ¿Cómo? Bogotá: ILSA, 2003. (pp. 259290).

SPENCER, H. The Principles of Sociology. Vol. I. New York: D. Appleton \& Company, 1912.

SQUELLA, A. Introducción al Derecho. Santiago de Chile: Editorial Jurídica de Chile, 2000. 
STICHWEH, R. On the Genesis of World Society: Innovations and Mechanisms. The Journal of Sociology and Social Anthropology, Vol. II, nº. 3, 1998. Die Weltgesellschaft. Soziologische Analysen. Frankfurt a. M.: Suhrkamp, 2000.

Evolutionary Theory and the Theory of World Society. Soziale Systeme, Vol. 13, pp. 528-543, 2007.

TEUBNER, G. Breaking Frames: Economic Globalization and the Emergence of Lex Mercatoria. European Journal of Social Theory, Vol. 5, nº. 2, pp. 199-217, 2002.

. La transformación del pluralismo jurídico global. In: GÓMEZJARA, C. (Ed.). El Derecho como Sistema Autopoiético de la Sociedad Global. Lima: Ara Editores, 2005. (pp. 113-133).

TOMASIC, R. The Quest for Theory in the Sociology of Law. Current Sociology, Vol. 33, no. 1, pp. 2-29, 1985.

UPRIMY, R., RODRÍGUEZ, C., \& GARCÍA VILLEGAS, M. Entre el protagonismo y la rutina: Análisis sociojurídico de la Justicia en Colombia. In: FIX-FIERRO, H., FRIEDMAN, L. \& PÉREZ PERDOMO, R. (Eds.). Culturas jurídicas latinas de Europa y América en tiempos de globalización. México D. F.: Universidad Nacional Autónoma de México, 2003, (pp. 231-303).

VALLE, A. La cultura jurídica interna. Análisis de ciertas notas sobre Chile. Anuario de Filosofía Jurídica y Social, n’. 19, pp. 87-109, 2001.

WEBER, M. Nachtrag zu dem Aufsatz über R. Stammlers „Überwindung““ der materialistischen Geschichtsauffassung. In: Weber, M. (Ed.). Gesammelte Aufsätze zur Wissenschaftslehre. Tübingen: Verlag von J. C. B. Mohr (Paul Siebeck), 1922a. (pp. 556-579).

. Wirtschaft und Gesellschaft. Tübingen: J.C.B. Mohr (Paul Siebeck), 1922b. 
WILLKE, H. Societal guidance through law. In: TEUBNER, G.; FEBBRAJO, A. (Eds.). State, Law and Economy as autopoietic systems. Milan: Ed. Giuffre, 1992. (pp. 354-387).

WITKER, J.; NATAREN, C. F. Tendencias actuales del diseño del proceso acusatorio en America Latina y Mexico. Mexico D. F.: Universidad Nacional Autonoma de Mexico, 2010.

Recebido em: 07/11/2011

Aprovado em: 30/01/2012 